\title{
Un Centre d'études des religions populaires
}

\section{Bernard Lacroix}

Volume 38, 1971

La genèse de la Société des Missions-Étrangères de la province de Québec

URI : https://id.erudit.org/iderudit/1007268ar

DOI : https://doi.org/10.7202/1007268ar

Aller au sommaire du numéro

Éditeur(s)

Les Éditions Historia Ecclesiæ Catholicæ Canadensis Inc.

ISSN

0318-6172 (imprimé)

1927-7067 (numérique)

Découvrir la revue

Citer cet article

Lacroix, B. (1971). Un Centre d'études des religions populaires. Sessions d'étude - Société canadienne d'histoire de l'Église catholique, 38, 88-94.

https://doi.org/10.7202/1007268ar

Tous droits réservés @ Les Éditions Historia Ecclesiæ Catholicæ Canadensis Inc., 1972
Ce document est protégé par la loi sur le droit d'auteur. L'utilisation des services d’Érudit (y compris la reproduction) est assujettie à sa politique d'utilisation que vous pouvez consulter en ligne.

https://apropos.erudit.org/fr/usagers/politique-dutilisation/ 
lecteurs, pour justement être mieux à même de répondre à son premier objectif qui est de "susciter l'intérêt pour l'histoire de l'Église "? $\mathrm{Ne}$ serait-il pas à souhaiter que les réunions annuelles deviennent véritablement les États généraux de l'histoire de l'Église canadienne servant à regrouper tous ceux, spécialistes et amateurs, que passionne notre passé religieux ? C'est un vœu que nous osons formuler.

Voilà les suggestions que nous avions à faire: nous prions la direction de la SCHEC de bien vouloir les prendre le plus tôt possible en considération.

Pierre Hurtubise, o.m.i., Université Saint-Paul, Ottawa, Ont.

\section{Un Centre d'études des religions populaires}

Ce n'est pas à une honorable société comme La Société canadienne $d^{\prime}$ Histoire de l'Église catholique qu'on voudrait faire la leçon. Mais la situation religieuse étant devenue ce qu'elle est présentement, au Canada français et plus spécialement au Québec, nous craignons malgré nous que soient à jamais dévalorisés, oubliés même, en même temps qu'écartés de la vie pratique, des rites, des attitudes, des croyances qui ont véhiculé certaines valeurs sans lesquelles notre "petit peuple», comme disait le chanoine Groulx, n'aurait sûrement pas survécu et qui l'ont fait tel, riche en agir, sans qu'il sache toujours pourquoi. Pour sa part le Centre d'études des religions populaires (C.E.R.P.) de l'Université de Montréal n'a d'autres intentions que d'identifier ces phénomènes religieux « populaires », quels qu'ils soient, en rassemblant, préservant et, si possible, conservant le meilleur grâce à des archives écrites et audio-visuelles.

Que je vous explique en premier lieu (I) le contexte dans lequel a été créé et évolue encore le C.E.R.P., (II) les objectifs de l'équipe; pour vous dire enfin (III) les urgences qui présentement retiennent davantage notre attention. Les échanges qui suivront permettront les précisions que cet exposé veut appeler plutôt qu'expliciter.

\section{I \\ Un contexte de religion d'Occident}

Le Centre d'études des religions populaires (C.E.R.P.) existe depuis 1967; il est présentement greffé à une institution de recherches interdisciplinaires, l'Institut d'Études médiévales, qui œuvre à l'Université de Montréal depuis 1942. Cet organisme qui s'est spécialisé surtout dans le champ mixte des études littéraires et religieuses du moyen âge occidental compte près de 50 publications scientifiques éditées conjointement à Montréal et à Paris, chez J. Vrin. C'est donc à dire que toute recherche s'y trouve en lieu sûr, d'autant plus que l'objectif de cet Institut scientifique est l'identification, à ses origines, de l'homme d'Occi- 
dent. La connaissance de l'homme d'occident dans un cadre d'études médiévales nous a conduit très vite au Canada français, au Québec en particulier. Nous sommes tous nés au moyen âge. Étudier le moyen âge, c'est nous " photographier ». Sans oublier l'avantage de connaître ses enfances pour expliquer la vie adulte que le $\mathrm{XX}^{\bullet}$ siècle nous impose avec ses nouveaux problèmes et ses crises.

Nous ne recommencerons pas la preuve de la genèse médiévale du Canada français (cf. Pourquoi aimer le moyen âge, Euvre des Tracts, 367 , Montréal, 16 p.) quand il suffit de parcourir tout simplement les douzaines de volumes de la collection "Archives de Folklore" de l'Université Laval pour nous retrouver aux $\mathrm{XIII}^{\bullet}$ et $\mathrm{XIV}^{\bullet}$ siècles. Le fait demeure en effet que ce Montréalais, ce Québécois, ce Canadien français, nous le voyons naître en France, en Angleterre. Rien ne nous sépare encore de cc passé où noüs àvons appris à parler, à conjuguer nos verbes, à écrire notre langue. Ni la Révolution française, qui a fait dévier la ligne d'hérédité médiévale en France, ni la Réforme qui a aussi écarté l'Angleterre d'un certain passé, ne nous auront directement atteints. On peut même se demander si au Québec en particulier, en Acadie aussi, l'héritage français médiéval ne se serait pas transmis plus purement, du moins plus directement, qu'ailleurs.

C'est ainsi que nous retrouvons encore aujourd'hui dans notre langage populaire des mots, une phonétique, qui rappellent le meilleur moyen âge français. Notre manière de prononcer le français qui remonte à l'ancien français, avec ces accents "latins ". V.g.:

$$
\begin{aligned}
& \text { premier : primo } \\
& \text { troisième : tertio } \\
& \text { médecin : medicus }
\end{aligned}
$$

Du même point de vue notre anthroponomie populaire montre que nous avons été étonnamment fidèles au moyen âge. Non, on ne saurait étre plus médiéval que s'appeler LEBEL... C'est même glorieux de descendre ainsi de Philippe LeBel. Lamarche, Lambert, Langlais, Lalande, Leduc, Lecomte, Legrand, Lemoine, Richer, Breton, Martin, Bernier! Tous nos Henri, Louis, Denis, Philippe, Hugues, Alexis, Arthur, Bertrand, Gérard, Edouard, Germain, Raoul, Guillaume. Ce sont ces noms que l'on retrouve dans les fabliaux, les chansons de gestes et surtout en historiographie médiévale latine et française.

Et tout ce que la toponomie comparée aurait à nous apprendre aussi ! Tant de filiations ! de répétitions ! Mont-Royal. L'Isle SainteCroix. L'Alverne. On n'ose même pas commencer les " preuves ", tant il est facile d'y penser rien qu'à voir les lieux, rien qu'à les entendre nommer. Du même point de vue, notre géographie religieuse comparée serait aussi éloquente. Tous ces noms de saints et de saintes partout! 
Cette même hérédité " médiévale » s'est incarnée dans des institutions qui ont fait vivre les franco-québécois: les universités, les collèges, les pensionnats, sans compter les programmes d'études, nos chartes papales et pontificales, l'importance de Monseigneur dans la vie cléricale, sa cour, sa royauté vestimentaire ... On pourrait étudier les survivances du régime seigneurial et y retrouver les habitudes du $\mathrm{XV}^{\bullet}$ siècle, comme on pourrait étudier certains rites d'église, certains gestes d'artisans...

Grâce à l'Institut d'études médiévales encore, le C.E.R.P. peut identifier toutes ces créations de la littérature religieuse populaire, la tradition orale en particulier, les contes, les légendes et les chansons. Tout nous porte à retrouver dans la réalité d'ici l'environnement culturel, l'intégration des coutumes, et le merveilleux médiéval.

Il va sans dire qu'à date l'approche historique de l'étude des phénomènes religieux populaires a été largement favorisée par cette affliation à un institut d'études anciennes. Cette affliation tient surtout au fait que nous voulons connaître les faits jusque dans leurs origines, examiner autant l'être de notre religion que son devenir depuis le $\mathrm{XIV}^{e}$ siècle. Cette méthode étant acquise, et souvent vérifiée par la suite, voudrait, cependant, et de plus en plus, profiter de l'expérience des autres sciences humaines et techniques, car, en ce qui a trait surtout au C.E.R.P. la connaissance de l'homme religieux étant toujours au premier plan, il est normal que nous voulions le saisir de la manière la plus intégrale possible. Nous nous intéressons de plus en plus aux méthodes sociologiques, psychologiques et surtout à celles de l'anthropologie culturelle.

Tel est le contexte dans lequel a été créé et évolue depuis 1967 le C.E.R.P.

II

BUTS et OBJECTIFS du C.E.R.P.

\section{A. Un peu d'histoire.}

Une douzaine de professeurs et d'étudiants se réunissent à Magog en 1967 en vue d'ouvrir un secteur d'étude scientifique de la foi popu. laire. Dès 1969, l'équipe qui se regroupe sans cesse et change selon les intérêts immédiats de la recherche, est spontanée; elle est sans président, sans directeur, sans charte, sans " règlements ". A peine un secrétariat pour assurer les échanges et l'animation. En 1970 nous consacrons une journée à la bibliographie et y invitons des spécialistes étrangers. Les Universités du Québec nous appuient, le Conseil des Arts subventionne. Puis, une autre journée d'études, le 16 octobre en 1971, à Québec. Cette fois, il s'agit de la réunion d'un groupe plus immé. 
diatement intéressé par le merveilleux religieux, le merveilleux québécois. La journée promise en 1972 portera sur l'imagerie religieuse québécoise et ses implications idéologiques.

Déjà nous percevons, à l'expérience, les grands objectifs GÉNÉRAUX du C.E.R.P.

\section{B. BUTS GÉNÉRAUX}

1. une meilleure connaissance de l'homme religieux occidental pour une meilleure connaissance de l'homme tout court.

2. A la lumière de l'histoire comparée des religions, apprendre à identifier le phénomène religieux local. Il ne s'agit pas tellement d'une connaissance par opposition (v.g. religion d'élite vs religion populaire) mais d'une connaissance mixte, par voies parallèles, parfois d'excroissances à partir d'un culte plus officiel, ou à partir d'un culte plus populaire promu "culte public ». Déjà nous avons noté que la religion d'élite est plus savante, plus discursive et plus rationaliste en général; elle favorise davantage la liberté, l'épanouissement personnel et la promotion sociale. Tandis que la religion populaire, liée à la terre, au cosmos, aux saisons, serait davantage domestique, collective, plus près aussi du quotidien vécu que de l'actualité reçue.

3. Vérifier l'héritage reçu du moyen âge et véhiculé surtout aux $\mathrm{XVII}^{\circ}$ et $\mathrm{XIX}^{\mathbf{e}}$ siècles de l'Europe au Canada français. Ceci nous conduira à prendre davantage conscience de l'originalité des phénomènes religieux catholiques du Canada français, surtout au Québec et en Acadie.

C. En ce qui a trait aux objectifs plus PARTICULIERS, nous ne sommes pas encore absolument fixés. Nous avons une liste "internationale de savants préoccupés des études de religion populaire. Nous avons aussi réussi à date quatre rencontres d'équipes variables selon des idéologies et centres d'intérêts.

Nous avons réussi à publier annuellement, depuis trois ans, quatre Cahiers d'études des religions populaires. Les Actes de nos colloques d'octobre, sous presse, seront bientôt distribués au public par les Presses Universitaires de Québec. D'autres projets sont à l'étude, dont la formation d'équipes locales, d'archives régionales, etc. Encore une fois, la période d'invention demeure. Il s'agit d'une cuvre qui doit faire son chemin au rythme que les faits lui imposeront.

\section{III}

Quant aux urgences dont nous parlions plus haut, il n'y a qu'un seul mot, à notre avis qui les résume toutes: l'archivistique. Nous entendons ce mot, nous du moins, dans son sens le plus vaste possible des 
premiers témoignages, recueils, monuments, bibliothèques, calendriers, inventaires, fichiers, généalogies, photocopies, microfilms, machines parlantes, video-cassettes, musées, cinémathèques et phototèques. etc.

Nous distinguerions trois niveaux d'action archivistique. A. Recherches et documentation proprement dites. B. Classification. C. Connaissance du document.

\section{Recherches et Documentation}

Il existe déjà au Canada français, voire jusqu'en Louisiane et dans les milieux franco-américains, des dépôts de textes et documents oraux, visuels ou écrits qui intéressent la religion populaire. Nous connaissons les initiatives des Musées Nationaux et Provinciaux, les Archives de Folklore de Québec en particulier. D'autres organismes encore. Mais le plus urgent reste la création d'archives spécialisées. Tout est à inventorier encore par enquêtes, voyages, tournées qui identifient le mobilier, les institutions, les lieux où survit la religion populaire. Nous rendons ici hommage à plusieurs communautés religieuses (Jésuites, Ursulines, Congrégation Notre-Dame, Sœurs grises, etc.) qui ont déjà leurs archivistes, leurs fonds, leurs musées. Conserver, c'est déjà créer.

Mais nous ne pouvons oublier en même temps tout ce qui est arrivé récemment à notre art populaire en général, à l'exploitation mercantile, aux églises tout à coup vidées sans discernement. Voilà pourquoi nous croyons de toute urgence rappeler à votre Société qu'elle a un devoir, au moins prévenir son public, afin que les témoignages et documents de notre vie religieuse traditionnelle ne prennent pas des routes a étrangères ». Faudrait-il par exemple créer au Québec une église-musée d'objets religieux traditionnels? Qui multipliera les enquêtes orales, visuelles, sur nos anciens rites, chants, croyances, livres et langages religieux, afin que les souvenirs ne soient pas perdus à jamais? Qui " enregistrera " le grégorien de nos chantres d'Église ? Et les vieux cantiques? Tant de possibilités nous sont offertes d'améliorer nos archives sonores et visuelles! De toute manière, c'est notre dernière chance de multiplier ces collections privées et publiques. J'ai remarqué - soit dit en passant - que beaucoup de jeunes avaient un sens inné de l'archivistique. L'occasion est à ne pas manquer, d'autant que les outils (machines parlantes, cassettes, photocopies, etc.) deviennent auprès d'eux de plus en plus " populaires ".

$$
-\mathbf{B}-
$$

L'autre niveau de travail archivistique que nous prévoyons est celui du catalogage et de la bibliographie. Après les inventaires, la juxta- 
position, la coordination. Ici encore les initiatives privées devront faire leur part dans la collection cataloguée du matériau de religion populaire. Nous conseillerions, pour l'instant, la multiplication de centres archivistiques (toujours au sens large du mot) régionaux et locaux plutôt qu'un seul Musée, ou qu'un seul lieu d'archives, sauvegardant les initiatives locales davantages actives en matières de religion populaire; il appartiendrait aux Archives centrales d'animer, d'encourager les initiatives, de penser aux guides, aux répertoires possibles, aux filmathèques, à la photocopie. Ainsi la classification n'étant jamais hâtive et prématurée, une entreprise archivistique bien animée plutôt que tota. lement absorbée par le Ministère des Affaires culturelles, resterait davantage collective et populaire. Pour aller au plus court, avant d'enfermer les jeunes dans un Musée pour y travailler durant les vacances, je les enverrais plutôt cueillir, "collectionner ", se fabriquer des archives privées. Les Archives Publiques auraient ainsi l'information nécessaire pour créer tout un mouvement de "sauvegarde".

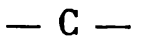

Quant à la connaissance du document (visuel, écrit ou oral), elle devra à notre avis respecter en tout premier lieu « texte et contexte ». C'est pour cette raison que nous accordons à la méthode historique dans l'étude du phénomène religieux, une priorité de fait et d'intention. Non que nous refusions les autres approches, mais nous estimons qu'au début d'une étude, la première politesse que nous nous devons d'accorder à un phénomène religieux, surtout s'il est populaire, est de l'inventorier, de l'identifier, de l'écouter, c'est-à-dire de le situer dans le temps autant que dans l'espace. Ceci, avant toute autre initiative.

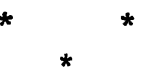

La Société canadienne d'histoire de l'Église catholique nous est apparue en définitive comme un lieu privilégié d'échanges et de projets quant à l'étude scientifique de la religion populaire en Amérique française. Ce que nous avons dit des premières études de religion populaire au Québec, et surtout de l'urgence archivistique, était en somme un moyen, bien limité j'en conviens, de répondre à votre confiance.

* BIBLIOGRAPHIE SOMMAIRE. Sur la religion populaire en Occident, nous recommandons les travaux de LeBras, Delaruelle, Gaïffier et Toussant, Rousset, et d'autres noms bien connus des médiévistes. Au Québec, quelques tentatives, éloquentes déjà, ont été pratiquées dans le Bulletin des Recherches historiques, sans oublier les bibliographies de Falardeau, Du Berger, Pierre Savard, etc. Les Actes de deux Colloques d'octobre 1970, 1971, publiés par les Presses de 
l'université Laval [1972] offrent au lecteur les précisions qui s'imposent.

Benoit LACRoIX, o.p.,

Institut d'études médiévales, Montréal.

\section{Les Instituts d'Histoire}

Qui oserait maintenant parler au nom de la dizaine d'Instituts d'Histoire existant au Québec si ce n'est en se restreignant au strict plan de la recherche. De ce point de vue, je crois représenter l'opinion de nombreux professeurs en voulant souligner les mérites des membres de la Société Canadienne d'Histoire de l'Église Catholique pour avoir organisé à chaque année depuis 1933, un colloque sur les problèmes d'histoire ảu catholicisme au Québec et publier leurs travaux dans un rapport annuel. D'ailleurs Jean-Charles Falardeau et Pierre Savard, soucieux de faire le point et de tracer d'autres avenues dans ce secteur si peu développé de la recherche ont déjà mentionné les avantages de ces publication ${ }^{1}$. Pour autant, il n'est pas moins vrai que les professeurs universitaires et les étudiants ne constituent pas l'assistance la plus nombreuse à ces colloques et que les publications annuelles ne se trouvent pas encore dans toutes les bibliothèques d'université. Faut-il expliquer cette situation par le manque d'intérêt pour l'histoire religieuse dont l'enseignement, jusqu'à tout récemment, était à peu près inexistant dans nos départements d'histoire ? Je suis enclin à accepter cette explication quoiqu'il ne faille pas se cacher que les universitaires ont souvent eu à redire à la qualité des travaux publiés. Certains trouvent inutiles ces monographies ou biographies, trop souvent élogieuses ou complaisantes, manquant parfois de rigueur intellectuelle, faisant fi de la longue durée pour y préférer la description chronologique et souvent anecdotique. S'il faut leur donner en partie raison et améliorer la qualité des publications, il n'est certes pas inutile de rappeler à ces critiques que l'analyse et la synthèse dont ils se réclament ne peuvent être réalisées que par la connaissance du quotidien anecdotique, du «banal » auquel il faut donner un sens. D'ailleurs Paul Leuilliot leur a déjà répondu en faisant sienne les paroles de Jal: "J'avoue, en toute humilité, que je suis de ceux qui se plaisent à la recherche de ces misères-là, comme les appellent nos grands esprits. J'ai la vue courte et le détail minuscule

1 Jean-Charles Falardeau, Recherches sociologiques, anthropologiques et psychulogiques sur la religion vécue au Canada français ", in Cahiers d'Etudes des Religions Populaires, vol. X, 1971, p. 4.21. «es recherches religieuses au Canada français ", in F. Dumont et Y. Martin, Situation de la recherche au Canada français, P.U.L., 1962, p. 209-229. Pierre SAvard, L'historien et la religion populaire au Canada français », in Cahiers d'Etudes des Religions Populaires, vol. 7, 1971, p. 29-39. 